\title{
Stress distribution of external hex joint implant-supported fixed prostheses in the posterior maxilla: 3-D finite element analysis
}

\section{ABSTRACT $n^{\circ} 15436$}

Authors :

\begin{tabular}{|c|c|l|}
\hline Name & Email & \multicolumn{1}{c|}{ Country } \\
\hline Yang Hongso ${ }^{*}$ & yhsdent@jnu.ac.kr & Korea, Republic of (South Korea) \\
\hline Kim Hyun-Ah & orion9136@naver.com & Korea, Republic of (South Korea) \\
\hline Chung Hyunju & hjchung@jnu.ac.kr & Korea, Republic of (South Korea) \\
\hline Park Jae-Ho & hugoism@naver.com & Korea, Republic of (South Korea) \\
\hline
\end{tabular}

Type of presentation : : Poster Display

Clinical Research - surgery : Basic research

I want to apply for a travel grant as : -

Responsible author last name : Yang

Supervisors Firstname : Hongso

If your research is accredited, please enter your accreditation number :

Keywords : Biomechanics, Finite elemente analysis, Morphometric analysis, Prosthodontics

Résumé [ 2280 Characters ]

\section{Background (500 characters maximum)}

If the bone graft is not performed in the atrophied posterior maxilla, there are various implant placement options, such as using a short implant or changing the direction of the implant placement to use more residual bone. Biomechanical information regarding different implant systems and different implant placement options is not sufficient to determine a reliable treatment plan and implant selection.

\section{Aim/Hypothesis (300 characters maximum)}

The purpose of this study was to analyze functional stresses generated in the external hex joint implant, prosthesis and supporting bone in the posterior maxilla using finite element method.

\section{Materials and Methods (1000 characters maximum)}

Two external hex type implant (Ø5 X 7mm, Ø5 X 13mm) with the design of the US3 SA fixture of Osstem (Seoul, Korea) were modeled by computer-aided design software (Solidwork 2018). The posterior maxillary bone and 3-unit all zirconia crowns were also simulated. Three different scenarios were modeled with various implant alignment (S3E; 3 unit fixed prosthesis on 3 short implant abutments, S2E; 3 unit fixed prosthesis on 2 short $(7 \mathrm{~mm})$ implant abutments, A2E; 3 unit fixed prosthesis on 1 short implant abutment and 1 long $(13 \mathrm{~mm})$ implant with an angled abutment). A load of $30 \mathrm{~N}$ was applied vertically and obliquely, respectively to each of 12 occlusal contact points of the crown. 


\section{Results (1000 characters maximum)}

Regardless of load direction, the highest von Mises stresses of the implants and prosthesis occurred in the screw tightening contact area of the external hex abutment. The values of maximum von Mises stresses due to vertical load were similar between each model. The values ranged from $250 \mathrm{MPa}$ to $270 \mathrm{MPa}$. The maximum von Mises stresses produced by the oblique load at the abutment were 277,283 , and $317 \mathrm{MPa}$ for the A2E, S3E, and S2E models, respectively. The maximum compressive stresses due to the oblique load on the marginal bone adjacent to the implant were $224 \mathrm{MPa}$ in S3E, $342 \mathrm{MPa}$ in A2E, and $362 \mathrm{MPa}$ in S2E, respectively.

\section{Conclusions and Clinical Implications (500 characters maximum)}

Externally connection design of implant showed well-distributed stress around all components of the implant system. The maximum stresses generated in the implant was within the yield strength limit of the material. A 3-unit fixed bridge with two short implants

(Model S2E) generated the largest compressive stresses around the bone adjacent to the external hex type implant.

\section{CONFLICTS OF INTEREST}

Interest link in the last three years, with the following companies :

Research support/Scientific studies: NON

Consultancy, Expert: NON

Trainings, Teaching: NON

Advertising documents: NON

Invitation to national or international congresses: NON

Stock shareholder: NON

Patent or product inventor:NON 